\title{
Real Hamiltonian Forms of Affine Toda Models Related to Exceptional Lie Algebras
}

\author{
Vladimir S. GERDJIKOV ${ }^{\dagger}$ and Georgi G. GRAHOVSKI ${ }^{\dagger \dagger}$ \\ $\dagger$ Institute for Nuclear Research and Nuclear Energy, Bulgarian Academy of Sciences, \\ 72 Tsarigradsko Chaussee, 1784 Sofia, Bulgaria \\ E-mail: gerjikov@inrne.bas.bg,grah@inrne.bas.bg \\ $\ddagger$ Laboratoire de Physique Théorique et Modélisation, Université de Cergy-Pontoise, \\ 2 Avenue Adolphe Chauvin, F-95302 Cergy-Pontoise Cedex, France
}

Received December 19, 2005, in final form February 05, 2006; Published online February 17, 2006

Original article is available at http://www.emis.de/journals/SIGMA/2006/Paper022/

\begin{abstract}
The construction of a family of real Hamiltonian forms (RHF) for the special class of affine $1+1$-dimensional Toda field theories (ATFT) is reported. Thus the method, proposed in [1] for systems with finite number of degrees of freedom is generalized to infinitedimensional Hamiltonian systems. The construction method is illustrated on the explicit nontrivial example of RHF of ATFT related to the exceptional algebras $\mathbf{E}_{6}$ and $\mathbf{E}_{7}$. The involutions of the local integrals of motion are proved by means of the classical $R$-matrix approach.
\end{abstract}

Key words: solitons; affine Toda field theories; Hamiltonian systems

2000 Mathematics Subject Classification: 37K15; 17B70; 37K10; 17B80

\section{Introduction}

To each simple Lie algebra rank $g$ one can relate Toda field theory (TFT) in $1+1$ dimensions. It allows Lax representation: $[L, M]=0$, where $L$ and $M$ are first order ordinary differential operators, see e.g. $[2,3,4,5,6,7]$ :

$$
\begin{aligned}
& L \psi \equiv\left(i \frac{d}{d x}-i q_{x}(x, t)-\lambda J_{0}\right) \psi(x, t, \lambda)=0, \quad q_{x}(x, t)=\sum_{k=1}^{r} q_{k, x} H_{k}, \\
& M \psi \equiv\left(i \frac{d}{d t}-\frac{1}{\lambda} I(x, t)\right) \psi(x, t, \lambda)=0 .
\end{aligned}
$$

whose potentials take values in $\mathfrak{g}$. Here $q(x, t) \in \mathfrak{h}$ is the Cartan subalgebra of $\mathfrak{g}, \vec{q}(x, t)=\sum_{k=1}^{r} q_{k} \vec{e}_{k}$ is its dual $r$-component vector, $r=$ rank $\mathfrak{g}$. $H_{k}$ are the Cartan generators dual to the orthonormal basis elements $\vec{e}_{k}$ in the root space, and

$$
J_{0}=\sum_{\alpha \in \pi} E_{\alpha}, \quad I(x, t)=\sum_{\alpha \in \pi} e^{-(\alpha, \vec{q}(x, t))} E_{-\alpha}
$$

By $\pi_{\mathfrak{g}}$ we denote the set of admissible roots of $\mathfrak{g}$, i.e. $\pi_{\mathfrak{g}}=\left\{\alpha_{0}, \alpha_{1}, \ldots, \alpha_{r}\right\}$ where $\alpha_{1}, \ldots, \alpha_{r}$ are the simple roots of $\mathfrak{g}$ and $\alpha_{0}$ is the minimal root of $\mathfrak{g}$. The corresponding TFT is known as the affine TFT. The Dynkin graph that corresponds to the set of admissible roots of $\mathfrak{g}$ is called 
extended Dynkin diagrams (EDD). The equations of motion are of the form:

$$
\frac{\partial^{2} \vec{q}}{\partial x \partial t}=\sum_{j=0}^{r} \alpha_{j} e^{-\left(\alpha_{j}, \vec{q}(x, t)\right)} .
$$

The present paper extends the ideas of [8] and [9] to the ATFT related to the exceptional simple Lie algebra $\mathbf{E}_{6}$; for finite Toda chains see [10, 11, 1, 12].

\section{The reduction group}

The operators $L$ and $M$ are invariant with respect to the reduction group $\mathcal{G}_{\mathbb{R}} \simeq \mathbb{D}_{h}$ where $h$ is the Coxeter number of $\mathfrak{g}$. It is generated by two elements satisfying $g_{1}^{h}=g_{2}^{2}=\left(g_{1} g_{2}\right)^{2}=\mathbb{1}$ which allow realizations both as elements in Aut $\mathfrak{g}$ and in Conf $\mathbb{C}$. The invariance condition has the form [2]:

$$
\begin{array}{ll}
C_{1}\left(U\left(x, t, \kappa_{1}(\lambda)\right)\right)=U(x, t, \lambda), & C_{1}\left(V\left(x, t, \kappa_{1}(\lambda)\right)\right)=V(x, t, \lambda), \\
C_{k}\left(U^{\dagger}\left(x, t, \kappa_{k}(\lambda)\right)\right)=U(x, t, \lambda), \quad & C_{k}\left(V^{\dagger}\left(x, t, \kappa_{k}(\lambda)\right)\right)=V(x, t, \lambda), \quad k=2,3 .
\end{array}
$$

where $U(x, t, \lambda)=-i q_{x}(x, t)-\lambda J_{0}$ and $V(x, t, \lambda)=-\frac{1}{\lambda} I(x, t)$. Here $C_{k}$ are automorphisms of finite order of $\mathfrak{g}$, i.e. $C_{1}^{h}=C_{2}^{2}=\left(C_{1} C_{2}\right)^{2}=\mathbb{1}$ while $\kappa_{k}(\lambda)$ are conformal mappings of the complex $\lambda$-plane:

$$
\kappa_{1}(\lambda)=\omega \lambda, \quad \kappa_{2}(\lambda)=\lambda^{*}, \quad \kappa_{3}(\lambda)=(\omega \lambda)^{*},
$$

where $\omega=\exp (2 \pi i / h)$. The algebraic constraints (2) are automatically compatible with the evolution. A number of nontrivial reductions of nonlinear evolution equations can be found in $[13,14]$.

\section{Spectral properties of the Lax operator}

The reduction conditions (2) lead to rather special properties of the operator $L$. Along with $L$ we will use also the equivalent system:

$$
\widetilde{L} m(x, t, \lambda) \equiv i \frac{d m}{d x}-i q_{x} m(x, t, \lambda)-\lambda\left[J_{0}, m(x, t, \lambda)\right]=0,
$$

where $m(x, t, \lambda)=\psi(x, t, \lambda) e^{i J_{0} x \lambda}$. Here $\vec{q}_{x}$ is the potential which we choose to be a Schwartztype function taking values in the complexified Cartan subalgebra $\mathfrak{h}_{\mathbb{C}} \subset \mathfrak{g}$. This means that the boundary conditions for $\vec{q}$ are determined up to the constant vector $\vec{\rho}_{0}$ :

$$
\vec{\rho}_{0}=\lim _{x \rightarrow \pm \infty} \vec{q}(x, t)
$$

The change of the variables $\vec{q}^{\prime}=\vec{q}-\vec{\rho}_{0}$ does not affect the potential and the spectral data of $\widetilde{L}$, but they change the right hand side of the ATFT equations into:

$$
\frac{\partial^{2} \vec{q}^{\prime}}{\partial x \partial t}=\sum_{j=0}^{r} s_{j} \alpha_{j} e^{-\left(\alpha_{j}, \vec{q}^{\prime}(x, t)\right)},
$$

where $s_{j}=\exp \left[-\left(\alpha_{j}, \vec{\rho}_{0}\right)\right]$ obviously satisfy the consistency conditions:

$$
\prod_{j=0}^{r} s_{j}^{n_{j}}=\exp \left(-\sum_{j=0}^{r}\left(n_{j} \alpha_{j}, \vec{\rho}_{0}\right)\right)=1 .
$$


where $n_{j}$ are the minimal positive integers for which $\sum_{j=0}^{r} n_{j} \alpha_{j}=0$. In the literature there are two canonical ways of fixing up the vector $\vec{\rho}_{0}$. The first one is to put $s_{j}=1$ for all $j$, the second is to have $s_{j}=n_{j}$ for all $j$.

The spectral properties of the Lax operator are rather involved due to the fact that both $J_{0}$ and $I(x, t)$ have complex-valued eigenvalues. In fact all these eigenvalues are constant and are proportional to $\omega^{k}, k=0,1, \ldots, h-1$, where $\omega=e^{2 \pi i / h}$ and $h$ is the Coxeter number of $\mathfrak{g}$. As a result one finds that the continuous spectrum of $L(\lambda)$ fills up $2 h$ rays passing through the origin:

$$
\lambda \in l_{\nu}: \arg \lambda=\frac{(\nu-1) \pi}{h} .
$$

For such operators one can introduce Jost solutions only for potentials on a compact support $[15,16]$ :

$$
\lim _{x \rightarrow \infty} \Psi(x, t, \lambda) e^{i \lambda J_{0} x+(i / \lambda) I_{0} t}=\mathbb{1}, \quad \lim _{x \rightarrow-\infty} \Phi(x, t, \lambda) e^{i \lambda J_{0} x+(i / \lambda) I_{0} t}=\mathbb{1},
$$

where

$$
I_{0}=\lim _{x \rightarrow \infty} I(x, t)=\sum_{k=0}^{r} E_{-\alpha_{k}}, \quad \alpha_{k} \in \pi(\mathfrak{g}) .
$$

Then the corresponding scattering matrix $T(t, \lambda)$ is defined by

$$
T(t, \lambda)=(\Psi(x, t, \lambda))^{-1} \Phi(x, t, \lambda) .
$$

The compactness of the potential ensures that the Jost solutions $\Phi(x, t, \lambda), \Psi(x, t, \lambda)$ and the scattering matrix $T(t, \lambda)$ are meromorphic functions of $\lambda$.

The fact that $\Phi(x, t, \lambda)$ and $\Psi(x, t, \lambda)$ are also Jost solutions of $M(\lambda)$ means that $T(t, \lambda)$ evolves according to:

$$
i \frac{d T}{d t}-\frac{1}{\lambda}\left[I_{0}, T(t, \lambda)\right]=0 .
$$

The limiting procedure to non-compact potentials can be done only for the so-called fundamental analytical solutions (FAS) $m_{\nu}(x, t, \lambda), \lambda \in \Omega_{\nu}$, i.e. $(\nu-1) \pi / h \leq \arg \lambda \leq \nu \pi / h$. Their construction is outlined in $[15,16]$ for generic complex-valued $J_{0}$. Our Lax pair is special, because it satisfies the reduction condition (2) with the Coxeter automorphism [17]:

$$
\bar{C}_{1}(X) \equiv C_{1}^{-1} X C_{1}, \quad C_{1}=e^{2 \pi i H_{\rho} / h}, \quad \rho=\frac{1}{2} \sum_{\alpha>0} \alpha
$$

obviously $C_{1}^{h}=\mathbb{1}$ and $\bar{C}_{1}\left(J_{0}\right)=\omega^{-1} J_{0}$. The FAS $m_{\nu}(x, t, \lambda)$ of $(3)$ are defined only in the sector $\Omega_{\ni}$ and satisfy:

$$
\bar{C}_{1}\left(m_{\nu}(x, t, \omega \lambda)\right)=m_{\nu-2}(x, t, \lambda), \quad \lambda \in l_{\nu-2} .
$$

Thus the inverse scattering problem for $L(\lambda)$ can be formulated as a Riemann-Hilbert problem for $m_{\nu}(x, t, \lambda)$ on the continuous spectrum $\Sigma \equiv \bigcup_{\nu=1}^{2 h} l_{\nu}$.

Skipping the details, we remark that equation (4) allows one to show that ATFT possess generating functionals of the integrals of motion. This can be shown easily if the potential $q(x, t)$ is such that $T(t, \lambda)$ can be diagonalized:

$$
T(t, \lambda)=u_{0}^{-1}(t, \lambda) D(\lambda) u_{0}(t, \lambda) .
$$


All factors in the above equation take values in the Lie group $\mathcal{G}$ with the Lie algebra $\mathfrak{g}$ and $D(\lambda)$ is a diagonal matrix. Then there exist a set of functions $\tau_{k}(\lambda), k=1, \ldots, r$ such that:

$$
D(\lambda)=\exp \left(\sum_{k=1}^{r} \frac{2 \tau_{k}(\lambda)}{\left(\alpha_{k}, \alpha_{k}\right)} H_{\alpha_{k}}\right),
$$

where $H_{\alpha_{k}}$ are the Cartan generators of $\mathfrak{g}$ corresponding to the simple root $\alpha_{k}$. Inserting (5) and (6) into (4) one finds that

$$
\frac{d D}{d t}=0, \quad \text { i.e. } \quad \frac{d \tau_{k}}{d t}=0
$$

for all $k=1, \ldots, r$. Obviously all eigenvalues of the scattering matrix $T(t, \lambda)$ will be expressed in terms of $\tau_{k}$. Each $\tau_{k}(\lambda)$ can be expanded over the negative powers of $\lambda$ :

$$
\tau_{k}(\lambda)=\sum_{s=0}^{\infty} I_{s}^{(k)} \lambda^{-s}
$$

and all the coefficients $I_{s}^{(k)}$ will be integrals of motion.

\section{Real Hamiltonian forms}

The Lax representations of the ATFT models (see e.g. $[2,3,4,18,6]$ and the references therein) are related mostly to the normal real form of the Lie algebra $\mathfrak{g}$, see [20].

Our aim here is to:

1) generalize the ATFT to complex-valued fields $\vec{q}^{\mathbb{C}}=\vec{q}^{0}+i \vec{q}^{1}$, and to

2) describe the family of RHF of these ATFT models.

We also provide a tool generalizing of the one in [1] for the construction of new inequivalent RHF's of the ATFT. The ATFT for the algebra $\operatorname{sl}(n)$ can be written down as an infinitedimensional Hamiltonian system as follows:

$$
\begin{aligned}
& \frac{d q_{k}}{d t}=\left\{q_{k}, H_{\mathrm{ATFT}}\right\}, \quad \frac{d p_{k}}{d t}=\left\{p_{k}, H_{\mathrm{ATFT}}\right\}, \\
& H_{\mathrm{ATFT}}=\int_{-\infty}^{\infty} d x\left(\frac{1}{2}(\vec{p}(x, t), \vec{p}(x, t))+\sum_{k=0}^{r} e^{-\left(\vec{q}(x, t), \alpha_{k}\right)}\right),
\end{aligned}
$$

where $\vec{q}(x, t)$ and $\vec{p}=\partial \vec{q} / \partial x$ are the canonical coordinates and momenta satisfying canonical Poisson brackets:

$$
\left\{p_{k}(x), q_{j}(y)\right\}=\delta_{j k} \delta(x-y) .
$$

Next we define the involution $\mathcal{C}$ acting on the phase space $\mathcal{M}$ as follows:

1) $\mathcal{C}\left(F\left(p_{k}, q_{k}\right)\right)=F\left(\mathcal{C}\left(p_{k}\right), \mathcal{C}\left(q_{k}\right)\right)$,

2) $\mathcal{C}\left(\left\{F\left(p_{k}, q_{k}\right), G\left(p_{k}, q_{k}\right)\right\}\right)=\{\mathcal{C}(F), \mathcal{C}(G)\}$

3) $\mathcal{C}\left(H\left(p_{k}, q_{k}\right)\right)=H\left(p_{k}, q_{k}\right)$.

Here $F\left(p_{k}, q_{k}\right), G\left(p_{k}, q_{k}\right)$ and the Hamiltonian $H\left(p_{k}, q_{k}\right)$ are functionals on $\mathcal{M}$ depending analytically on the fields $q_{k}(x, t)$ and $p_{k}(x, t)$. 
The complexification of the ATFT is rather straightforward. The resulting complex ATFT (CATFT) can be written down as standard Hamiltonian system with twice as many fields $\vec{q}^{a}(x, t), \vec{p}^{a}(x, t), a=0,1$ :

$$
\begin{aligned}
& \vec{p}^{\mathbb{C}}(x, t)=\vec{p}^{0}(x, t)+i \vec{p}^{1}(x, t), \quad \vec{q}^{\mathbb{C}}(x, t)=\vec{q}^{0}(x, t)+i \vec{q}^{1}(x, t), \\
& \left\{p_{k}^{0}(x, t), q_{j}^{0}(y, t)\right\}=-\left\{p_{k}^{1}(x, t), q_{j}^{1}(y, t)\right\}=\delta_{k j} \delta(x-y) .
\end{aligned}
$$

The densities of the corresponding Hamiltonian and symplectic form equal

$$
\begin{aligned}
& \mathcal{H}_{\mathrm{ATFT}}^{\mathbb{C}} \equiv \operatorname{Re} \mathcal{H}_{\mathrm{ATFT}}\left(\vec{p}^{0}+i \vec{p}^{1}, \vec{q}^{0}+i \vec{q}^{1}\right) \\
& \quad=\frac{1}{2}\left(\vec{p}^{0}, \vec{p}^{0}\right)-\frac{1}{2}\left(\vec{p}^{1}, \vec{p}^{1}\right)+\sum_{k=0}^{r} e^{-\left(\vec{q}^{0}, \alpha_{k}\right)} \cos \left(\left(\vec{q}^{1}, \alpha_{k}\right)\right), \\
& \omega^{\mathbb{C}}=\left(d \vec{p}^{0} \wedge d \vec{q}^{0}\right)-\left(d \vec{p}^{1},{ }_{,} \vec{q}^{1}\right) .
\end{aligned}
$$

The family of RHF then are obtained from the CATFT by imposing an invariance condition with respect to the involution $\tilde{\mathcal{C}} \equiv \mathcal{C} \circ *$ where by $*$ we denote the complex conjugation. The involution $\tilde{\mathcal{C}}$ splits the phase space $\mathcal{M}^{\mathbb{C}}$ into a direct sum $\mathcal{M}^{\mathbb{C}} \equiv \mathcal{M}_{+}^{\mathbb{C}} \oplus \mathcal{M}_{-}^{\mathbb{C}}$ where

$$
\mathcal{M}_{+}^{\mathbb{C}}=\mathcal{M}_{0} \oplus i \mathcal{M}_{1}, \quad \mathcal{M}_{-}^{\mathbb{C}}=i \mathcal{M}_{0} \oplus \mathcal{M}_{1},
$$

The phase space of the RHF is $\mathcal{M}_{\mathbb{R}} \equiv \mathcal{M}_{+}^{\mathbb{C}}$. By $\mathcal{M}_{0}$ and $\mathcal{M}_{1}$ we denote the eigensubspaces of $\mathcal{C}$, i.e. $\mathcal{C}\left(u_{a}\right)=(-1)^{a} u_{a}$ for any $u_{a} \in \mathcal{M}_{a}$.

Then extracting of Real Hamiltonian forms (RHF's) is similar to the obtaining a real forms of a semi-simple Lie algebra. The Killing form for the later is indefinite in general (it is negativelydefinite for the compact real forms). So one should not be surprised of getting RHF's with indefinite kinetic energy quadratic form. Of course this is an obstacle for their quantization.

Thus to each involution $\mathcal{C}$ satisfying 1)-3) one can relate a RHF of the ATFT. Due to the condition 3) $\mathcal{C}$ must preserve the system of admissible roots of $\mathfrak{g}$; such involutions can be constructed from the $\mathbb{Z}_{2}$-symmetries of the extended Dynkin diagrams of $\mathfrak{g}$ studied in [6].

\section{Examples}

In this Section we provide several examples of RHF of ATFT related to exceptional Kac-Moody algebras with height 1 . We show that the involutions $\mathcal{C}$ in all these cases are dual to $\mathbb{Z}_{2^{-}}$ symmetries of the extended Dynkin diagrams derived in [6].

The examples below illustrate the procedure outlined above and display new types of ATFT.

\section{$5.1 \quad \mathrm{E}_{6}^{(1)}$ Toda field theories}

The set of admissible roots for this algebra is

$$
\begin{aligned}
& \alpha_{1}=\frac{1}{2}\left(e_{1}-e_{2}-e_{3}-e_{4}-e_{5}-e_{6}-e_{7}+e_{8}\right), \quad \alpha_{2}=e_{1}+e_{2}, \\
& \alpha_{3}=e_{2}-e_{1}, \quad \alpha_{4}=e_{3}-e_{2}, \quad \alpha_{5}=e_{4}-e_{3}, \quad \alpha_{6}=e_{5}-e_{4}, \\
& \alpha_{0}=-\frac{1}{2}\left(e_{1}+e_{2}+e_{3}+e_{4}+e_{5}-e_{6}-e_{7}+e_{8}\right),
\end{aligned}
$$

where $\alpha_{1}, \ldots, \alpha_{6}$ form the set of simple roots of $\mathbf{E}_{6}$ and $\alpha_{0}$ is the minimal root of the algebra. This is the standard definition of the root system of $\mathbf{E}_{6}$ embedded into the 8-dimensional Euclidean space $\mathbb{E}^{8}$. The root space $\mathbb{E}_{6}$ of the algebra $\mathbf{E}_{6}$ is the 6 -dimensional subspace of $\mathbb{E}^{8}$ orthogonal to 
the vectors $e_{7}+e_{8}$ and $e_{6}+e_{7}+2 e_{8}$. Thus any vector $\vec{q}$ belonging to $\mathbb{E}_{6}$ has only 6 independent coordinates and can be written as:

$$
\vec{q}=\sum_{k=1}^{5} q_{k} e_{k}+q_{6} e_{6}^{\prime}, \quad e_{6}^{\prime}=\frac{1}{\sqrt{3}}\left(e_{6}+e_{7}-e_{8}\right) .
$$

Let us fix up the action of the involution $\mathcal{C}$ on a generic vector $\vec{q}$ in $\mathbb{E}^{8}$ by:

$$
\begin{aligned}
\mathcal{C}\left(q_{k}\right) & =-q_{5-k}+\frac{1}{2} \sum_{m=1}^{4} q_{m}, \quad \text { for } \quad k=1, \ldots, 4, \\
& =q_{13-k}-\frac{1}{2} \sum_{m=5}^{8} q_{m}, \quad \text { for } \quad k=5, \ldots, 8 .
\end{aligned}
$$

This action is compatible with the $\mathbb{Z}_{2}$-symmetry $\mathcal{C}^{\#}$ of the extended Dynkin diagram (see Fig. 1) and reflects an involution of the Kac-Moody algebra $\mathbf{E}_{6}^{(1)}$, see [21]. It acts on the root space as follows:

$$
\begin{aligned}
& \mathcal{C}^{\#} e_{k}=-e_{5-k}+\frac{1}{2} \sum_{m=1}^{4} e_{m}, \quad \text { for } k=1, \ldots, 4, \\
&=e_{13-k}-\frac{1}{2} \sum_{m=5}^{8} e_{m}, \quad \text { for } k=5, \ldots, 8, \\
& \mathcal{C}^{\#} \alpha_{1}=\alpha_{6}, \quad \mathcal{C}^{\#} \alpha_{3}=\alpha_{5}, \quad \mathcal{C}^{\#} \alpha_{k}=\alpha_{k}, \quad k=0,2,4 . \\
& \underbrace{}_{\alpha_{0}}
\end{aligned}
$$

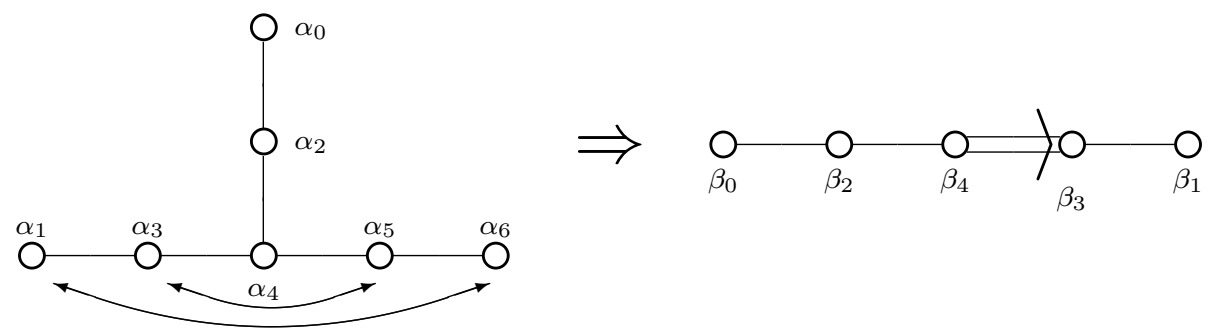

Figure 1. $\mathbf{E}_{6}^{(1)} \rightarrow \mathbf{F}_{4}^{(1)}$.

The involution $\mathcal{C}^{\#}$ splits the root space $\mathbb{E}_{6}$ into a direct sum of its eigensubspaces: $\mathbb{E}_{6}=$ $\mathbb{E}_{+} \oplus \mathbb{E}_{-}$with $\operatorname{dim} \mathbb{E}_{+}=4, \operatorname{dim} \mathbb{E}_{-}=2$. The vectors:

$$
\begin{aligned}
& \widetilde{e}_{1}=\frac{1}{2}\left(e_{5}-\sqrt{3} e_{6}^{\prime}\right), \quad \widetilde{e}_{2}=\frac{1}{2}\left(e_{1}+e_{2}+e_{3}+e_{4}\right), \quad \widetilde{e}_{3}=\frac{1}{2}\left(-e_{1}-e_{2}+e_{3}+e_{4}\right), \\
& \widetilde{e}_{4}=\frac{1}{2}\left(-e_{1}+e_{2}-e_{3}+e_{4}\right), \quad \widetilde{e}_{5}=\frac{1}{2}\left(-e_{1}+e_{2}+e_{3}-e_{4}\right), \quad \widetilde{e}_{6}=\frac{1}{2}\left(\sqrt{3} e_{5}+e_{6}^{\prime}\right) .
\end{aligned}
$$

form an orthonormal basis in $\mathbb{E}_{6}$. The first four satisfy $\mathcal{C}^{\#} \widetilde{e}_{k}=\widetilde{e}_{k}, k=1, \ldots, 4$, so they span $\mathbb{E}_{+}$; the last two span $\mathbb{E}_{-}$because $\mathcal{C}^{\#} \widetilde{e}_{j}=-\widetilde{e}_{j}, j=5,6$. In terms of $\widetilde{e}_{k}$ the admissible root system of $\mathbf{F}_{4}^{(1)}$ takes the standard form:

$$
\begin{aligned}
& \beta_{0}=-\widetilde{e}_{2}-\widetilde{e}_{1}, \quad \beta_{1}=\frac{1}{2}\left(\widetilde{e}_{1}-\widetilde{e}_{2}-\widetilde{e}_{3}-\widetilde{e}_{4}\right), \\
& \beta_{2}=\widetilde{e}_{2}-\widetilde{e}_{3}, \quad \beta_{3}=\widetilde{e}_{4}, \quad \beta_{4}=\widetilde{e}_{3}-\widetilde{e}_{4} .
\end{aligned}
$$

satisfying $\beta_{0}+2 \beta_{1}+2 \beta_{2}+4 \beta_{3}+3 \beta_{4}=0$. 
Let us take the complex vector $\vec{q}(x, t)=\vec{q}^{0}(x, t)+i \vec{q}^{1}(x, t) \in \mathbb{E}_{6}$ (i.e., of the form (8)) and let $\vec{p}(x, t)=\partial \vec{q} / \partial x$. Let us denote their projections onto $\mathbb{E}_{ \pm}$by $\vec{q}_{ \pm}$and $\vec{p}_{ \pm}$respectively. Then the densities $\mathcal{H}_{1}^{\mathbb{R}}, \omega_{1}^{\mathbb{R}}$ for the RHF of AFTF equal:

$$
\begin{aligned}
\mathcal{H}_{1}^{\mathbb{R}}= & \frac{1}{2}\left(\left(\vec{p}_{+}^{0}(x, t), \vec{p}_{+}^{0}(x, t)\right)-\left(\vec{p}_{-}^{0}(x, t), \vec{p}_{-}^{0}(x, t)\right)\right)+\mathrm{e}^{-\left(\vec{q}_{+}^{0}(x, t), \beta_{0}\right)} \\
& +2 \mathrm{e}^{-\left(\vec{q}_{+}^{0}(x, t), \beta_{1}\right)} \cos \left(\left(\vec{q}_{-}^{1}(x, t), \widetilde{e}_{5}+\sqrt{3} \widetilde{e}_{6}\right)\right)+2 \mathrm{e}^{-\left(\vec{q}_{+}^{0}(x, t), \beta_{2}\right)} \\
& +4 \mathrm{e}^{-\left(\vec{q}_{+}^{0}(x, t), \beta_{3}\right)} \cos \left(\left(\vec{q}_{-}^{1}(x, t), \widetilde{e}_{5}\right)\right)+3 \mathrm{e}^{-\left(\vec{q}_{+}^{0}(x, t), \beta_{4}\right)}, \\
\omega_{1}^{\mathbb{R}}= & \left(\delta \vec{p}_{+}(x) \wedge \delta \vec{q}_{+}(x)\right)-\left(\delta \vec{p}_{-}(x) \bigwedge_{1} \delta \vec{q}_{-}(x)\right),
\end{aligned}
$$

If we put $\vec{q}_{-}(x, t)=0$ then also $\vec{p}_{-}(x, t)=0$ and we get the reduced ATFT related to the Kac-Moody algebra $\mathbf{F}_{4}^{(1)}[6]$.

\section{$5.2 \quad \mathrm{E}_{7}^{(1)}$ Toda field theories}

The set of admissible roots for this algebra is

$$
\begin{aligned}
& \alpha_{1}=\frac{1}{2}\left(e_{1}-e_{2}-e_{3}-e_{4}-e_{5}-e_{6}-e_{7}+e_{8}\right), \quad \alpha_{2}=e_{1}+e_{2}, \\
& \alpha_{3}=e_{2}-e_{1}, \quad \alpha_{4}=e_{3}-e_{2}, \quad \alpha_{5}=e_{4}-e_{3}, \quad \alpha_{6}=e_{5}-e_{4}, \\
& \alpha_{7}=e_{6}-e_{5}, \quad \alpha_{0}=e_{7}-e_{8},
\end{aligned}
$$

where $\alpha_{1}, \ldots, \alpha_{7}$ form the set of simple roots of $\mathbf{E}_{7}$ and $\alpha_{0}$ is the minimal root of the algebra. This is the standard definition of the root system of $\mathbf{E}_{7}$ embedded into the 8-dimensional Euclidean space $\mathbb{E}^{8}$. The root space $\mathbb{E}_{7}$ of the algebra $\mathbf{E}_{7}$ is the 7-dimensional subspace of $\mathbb{E}^{8}$ orthogonal to the vector $e_{7}+e_{8}$. Thus any vector $\vec{q}$ belonging to $\mathbb{E}_{7}$ has 7 independent coordinates and can be written as:

$$
\vec{q}=\sum_{k=1}^{6} q_{k} e_{k}+q_{7} e_{7}^{\prime}, \quad e_{7}^{\prime}=\frac{1}{\sqrt{2}}\left(e_{7}-e_{8}\right) .
$$

Let us fix up the action of the involution $\mathcal{C}$ on a generic vector $\vec{q}$ in $\mathbb{E}^{8}$ by equation (9). This action is compatible with the $\mathbb{Z}_{2}$-symmetry $\mathcal{C}^{\#}$ of the extended Dynkin diagram (see Fig. 2) and reflects an involution of the Kac-Moody algebra $\mathbf{E}_{7}^{(1)}$, see [21]. It acts on the root space as in equation (10) above.

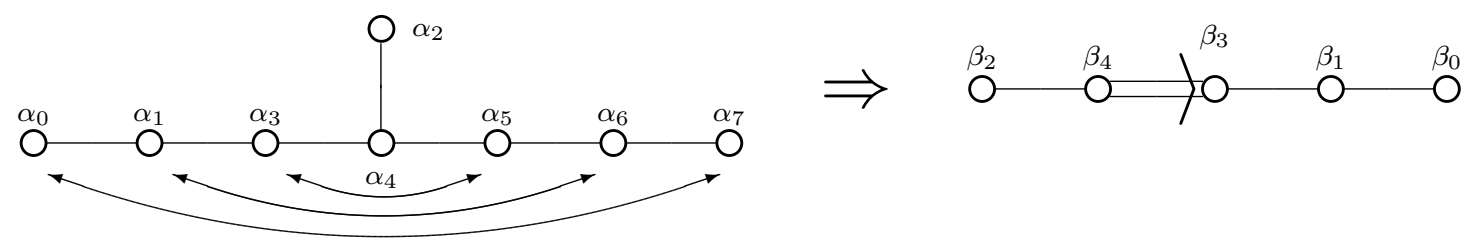

Figure 2. The $\mathbf{E}_{7}^{(1)} \rightarrow \mathbf{E}_{6}^{(2)}$ RHF of affine TFT.

However its action on the root space $\mathbb{E}_{7}$ is different. It splits $\mathbb{E}_{7}$ into a direct sum of its eigensubspaces: $\mathbb{E}_{7}=\mathbb{E}_{+} \oplus \mathbb{E}_{-}$with $\operatorname{dim} \mathbb{E}_{+}=4, \operatorname{dim} \mathbb{E}_{-}=3$. The vectors:

$$
\begin{aligned}
& \widetilde{e}_{1}=\frac{1}{2 \sqrt{2}}\left(e_{1}+e_{2}+e_{3}+e_{4}+e_{5}-e_{6}-\sqrt{2} e_{7}^{\prime}\right), \quad \widetilde{e}_{3}=\frac{1}{\sqrt{2}}\left(-e_{1}+e_{4}\right), \\
& \widetilde{e}_{2}=\frac{1}{2 \sqrt{2}}\left(-e_{1}-e_{2}-e_{3}-e_{4}+e_{5}-e_{6}-\sqrt{2} e_{7}^{\prime}\right), \quad \widetilde{e}_{4}=\frac{1}{\sqrt{2}}\left(-e_{2}+e_{3}\right),
\end{aligned}
$$


form an orthonormal basis in $\mathbb{E}_{+}$. Indeed, they satisfy $\left(\widetilde{e}_{j}, \widetilde{e}_{k}\right)=\delta_{j k}$ and $\mathcal{C}^{\#} \widetilde{e}_{k}=\widetilde{e}_{k}, k=1, \ldots, 4$. In terms of $\widetilde{e}_{k}$ the admissible root system of $\mathbf{E}_{6}^{(2)}$ takes the standard form:

$$
\begin{aligned}
& \beta_{0}=-\widetilde{e}_{2}-\widetilde{e}_{1}, \quad \beta_{1}=\widetilde{e}_{2}-\widetilde{e}_{3}, \quad \beta_{2}=\widetilde{e}_{3}-\widetilde{e}_{4}, \\
& \beta_{3}=2 \widetilde{e}_{4}, \quad \beta_{4}=\widetilde{e}_{1}-\widetilde{e}_{2}-\widetilde{e}_{3}-\widetilde{e}_{4},
\end{aligned}
$$

satisfying $\beta_{0}+2 \beta_{1}+\beta_{2}+3 \beta_{3}+2 \beta_{4}=0$.

The vectors

$$
\widetilde{e}_{5}=\frac{1}{2}\left(-e_{1}+e_{2}+e_{3}-e_{4}\right), \quad \widetilde{e}_{6}=\frac{1}{\sqrt{2}}\left(e_{5}+e_{6}\right), \quad \widetilde{e}_{7}=\frac{1}{2}\left(-e_{5}+e_{6}-\sqrt{2} e_{7}^{\prime}\right),
$$

span $\mathbb{E}_{-}$because $\mathcal{C}^{\#} \widetilde{e}_{j}=-\widetilde{e}_{j}, j=5,6,7$.

Let us take the complex vector $\vec{q}(x, t)=\vec{q}^{0}(x, t)+i \vec{q}^{1}(x, t) \in \mathbb{E}_{7}$ (i.e., of the form (11)) and let $\vec{p}(x, t)=\partial \vec{q} / \partial x$. Let us denote their projections onto $\mathbb{E}_{ \pm}$by $\vec{q}_{ \pm}$and $\vec{p}_{ \pm}$respectively. Then the densities $\mathcal{H}_{1}^{\mathbb{R}}, \omega_{1}^{\mathbb{R}}$ for the RHF of AFTF equal:

$$
\begin{aligned}
\mathcal{H}_{2}^{\mathbb{R}}= & \frac{1}{2}\left(\left(\vec{p}_{+}^{0}(x, t), \vec{p}_{+}^{0}(x, t)\right)-\left(\vec{p}_{-}^{0}(x, t), \vec{p}_{-}^{0}(x, t)\right)\right)+2 \mathrm{e}^{-\left(\vec{q}_{+}^{0}(x, t), \beta_{0}\right)} \cos \left(\left(\vec{q}_{-}^{1}(x, t), \widetilde{e}_{7}\right)\right) \\
& +4 \mathrm{e}^{-\left(\vec{q}_{+}^{0}(x, t), \beta_{1}\right)} \cos \left(\left(\vec{q}_{-}^{1}(x, t), \frac{1}{2}\left(\widetilde{e}_{5}+\sqrt{2} \widetilde{e}_{6}-\widetilde{e}_{7}\right)\right)+2 \mathrm{e}^{-\left(\vec{q}_{+}^{0}(x, t), \beta_{2}\right)}\right. \\
& +6 \mathrm{e}^{-\left(\vec{q}_{+}^{0}(x, t), \beta_{3}\right)} \cos \left(\left(\vec{q}_{-}^{1}(x, t), \widetilde{e}_{5}\right)\right)+4 \mathrm{e}^{-\left(\vec{q}_{+}^{0}(x, t), \beta_{4}\right)}, \\
\omega_{2}^{\mathbb{R}}= & \left(\delta \vec{p}_{+}(x) \wedge \delta \vec{q}_{+}(x)\right)-\left(\delta \vec{p}_{-}(x) \wedge \delta \vec{q}_{-}(x)\right) .
\end{aligned}
$$

Again, if we put $\vec{q}_{-}(x, t)=0$ then also $\vec{p}_{-}(x, t)=0$ and we get the reduced ATFT related to the Kac-Moody algebra $\mathbf{E}_{6}^{(2)}[6]$.

\section{Classical $R$-matrix method and ATFT}

There are several methods to approach the Hamiltonian properties of the ATFT, see e.g. [10, $18,13,11,19]$. One of the effective methods is based on the well known classical $R$-matrix [22] which is introduced by:

$$
\{U(x, \lambda) \underset{,}{\otimes} U(y, \mu)\}=[R(\lambda, \mu), U(x, \lambda) \otimes \mathbb{1}+\mathbb{1} \otimes U(y, \mu)] \delta(x-y),
$$

where $\{U(x, \lambda) \underset{,}{\otimes} U(y, \mu)\}_{i j, k l}=\left\{U_{i j}(x, \lambda), U_{k l}(y, \mu)\right\}$. In order to apply this definition effectively we make use of another Lax operator for the ATFT:

$$
\begin{aligned}
& \widetilde{\widetilde{L}}(x, \lambda) \equiv i \frac{d \widetilde{\widetilde{\psi}}}{d x}+U(x, \lambda) \widetilde{\widetilde{\psi}}(x, \lambda)=0, \\
& U(x, \lambda)=-\frac{i}{2} \sum_{j=1}^{r} q_{j, x} H_{j}-\lambda \sum_{j=0}^{r} e^{-\left(\alpha_{j}, \vec{q}\right) / 2} E_{\alpha_{j}},
\end{aligned}
$$

which is gauge equivalent to $L(1)$. Since the Poisson brackets are introduced by (7) the left hand side of (12) takes the form:

$$
\left\{U_{i j}(x, \lambda) \underset{,}{\otimes} U_{k l}(y, \mu)\right\}=\frac{i}{4} \sum_{k=0}^{r} e^{-\left(\alpha_{k}, \vec{q}\right) / 2}\left(\mu H_{\alpha_{k}} \otimes E_{\alpha_{k}}-\lambda E_{\alpha_{k}} \otimes H_{\alpha_{k}}\right) \delta(x-y) .
$$


Thus equation (12) becomes an over-determined set of equations for $R(\lambda, \mu)$ which is solved by [22]:

$$
\begin{aligned}
R(\lambda, \mu) & =\frac{1}{4 i} \frac{\lambda^{h}+\mu^{h}}{\lambda^{h}-\mu^{h}} \sum_{k=1}^{r} H_{k} \otimes H_{k}+\frac{1}{2 i} \sum_{\alpha \in \Delta}\left(\frac{\lambda}{\mu}\right)^{p(\alpha)} \frac{\mu^{h} E_{\alpha} \otimes E_{-\alpha}}{\lambda^{h}-\mu^{h}} \\
& =\frac{1}{4 i \sinh (h \eta / 2)}\left(\cosh (h \eta / 2) \sum_{k=1}^{r} H_{k} \otimes H_{k}+\sum_{\alpha \in \Delta} e^{(p(\alpha)-h / 2) \eta} E_{\alpha} \otimes E_{-\alpha}\right),
\end{aligned}
$$

where $h$ is the Coxeter number of $\mathfrak{g}, \eta=\ln (\lambda / \mu)$ and $p(\alpha)$ is the height of the root $\alpha$ modulo $h$. If $\alpha=\sum_{j=1}^{r} n_{\alpha, j} \alpha_{j}$ where $n_{\alpha, j}$ are integers, then $p(\alpha)=\sum_{j=1}^{r} n_{\alpha, j}$.

The relation (12) allows one to derive the Poisson brackets between the matrix elements of the fundamental solutions $T_{x_{0}}^{+}(x, \lambda)$ and $T_{x_{0}}^{-}(x, \lambda)$ (or the scattering matrix $T_{x_{0}}(\lambda)$ ) of $L$ which are defined by:

$$
\begin{aligned}
& L T_{x_{0}}^{ \pm}(x, \lambda)=0, \quad \lim _{x \rightarrow \pm x_{0}} T_{x_{0}}^{ \pm}(x, \lambda) e^{-i \lambda x J_{0}}=\mathbb{1}, \\
& T_{x_{0}}(\lambda)=\left(T_{x_{0}}^{+}(x, \lambda)\right)^{-1} T_{x_{0}}^{-}(x, \lambda) .
\end{aligned}
$$

Then

$$
\left\{T_{x_{0}}^{ \pm}(x, \lambda) \underset{,}{\otimes} T_{x_{0}}^{ \pm}(y, \mu)\right\}=\left[R(\lambda, \mu), T_{x_{0}}^{ \pm}(x, \lambda) \otimes T_{x_{0}}^{ \pm}(y, \mu)\right]
$$

and

$$
\left\{T_{x_{0}}(\lambda) \underset{,}{\otimes} T_{x_{0}}(\mu)\right\}=\left[R(\lambda, \mu), T_{x_{0}}(\lambda) \otimes T_{x_{0}}(\mu)\right]
$$

These results hold true for potentials on compact support provided we choose $x_{0}$ large enough so that $\vec{q}_{x}=0$ for $|x|>x_{0}$.

With $T_{x_{0}}(t, \lambda)$ we can associate a set of generating functionals $\tau_{k}\left(x_{0}, \lambda\right)$ of the integrals of motion

$$
T_{x_{0}}(t, \lambda)=u_{0}^{-1}\left(x_{0}, t, \lambda\right) D_{x_{0}}(\lambda) u_{0}\left(x_{0}, t, \lambda\right), \quad D_{x_{0}}(\lambda)=\exp \left(\sum_{k=1}^{r} \frac{2 \tau_{k}\left(x_{0}, \lambda\right)}{\left(\alpha_{k}, \alpha_{k}\right)} H_{\alpha_{k}}\right) .
$$

Again we can write the expansion

$$
\tau_{k}\left(x_{0}, \lambda\right)=\sum_{s=0}^{\infty} I_{x_{0}, k}^{(s)} \lambda^{-s}
$$

An important consequence of (13) is that the functions $\tau_{k}\left(x_{0}, \lambda\right)$ are in involution. Indeed from (13) there follows that:

$$
\left\{\operatorname{tr} T_{x_{0}}^{k}(\lambda), \operatorname{tr} T_{x_{0}}^{p}(\mu)\right\}=0
$$

for any pair of integers $k, p$. Obviously $\operatorname{tr} T^{k}(\lambda)$ can be expressed in terms of the invariants $\tau_{k}(\lambda)(6)$ of the scattering matrix $T(\lambda) \in \mathcal{G}$. Therefore from (14) we find that

$$
\left\{\tau_{k}\left(x_{0}, \lambda\right), \tau_{m}\left(x_{0}, \mu\right)\right\}=0, \quad 1 \leq k, m \leq r,
$$

i.e. the integrals of motion $I_{k}^{(s)}$ are all in involution:

$$
\left\{I_{x_{0}, k}^{(s)}, I_{x_{0}, m}^{(n)}\right\}=0, \quad 0 \leq k, m \leq r, \quad s, n \geq 0 .
$$


In order to derive the corresponding results for the RHF of the ATFT we have to use the fact that the automorphism $\mathcal{C}$ induces an automorphism $\mathcal{C}^{\vee}$ on the Lie algebra $\mathfrak{g}$ and on the Lax operator as follows [23]:

$$
\mathcal{C}^{\vee}\left(H_{j}\right)=H_{\mathcal{C}^{\#}\left(e_{j}\right)}, \quad \mathcal{C}^{\vee} E_{\alpha}=E_{\mathcal{C}^{\#}(\alpha)}, \quad L\left(\mathcal{C}\left(\vec{q}^{*}\right), x, \lambda^{*}\right)=\mathcal{C}^{\vee}(L(\vec{q}, x, \lambda))^{*}
$$

These relations allow one to prove that the fundamental solution $T_{x_{0}}(x, \lambda)$ and the scattering matrix $T_{x_{0}}(\lambda)$ for the corresponding RHF model has the properties:

$$
\left(T_{x_{0}}\left(x, \lambda^{*}\right)\right)^{*}=\mathcal{C}^{\vee}\left(T_{x_{0}}(x, \lambda)\right), \quad\left(T_{x_{0}}\left(\lambda^{*}\right)\right)^{*}=\mathcal{C}^{\vee}\left(T_{x_{0}}(\lambda)\right),
$$

and as a consequence: $\left.\left(\tau_{k}\left(\lambda^{*}\right)\right)^{*}=\tau_{\bar{k}}(\lambda)\right)$, where $\bar{k}$ is defined through $\mathcal{C}^{\#}\left(\alpha_{k}\right)=\alpha_{\bar{k}}$.

\section{Conclusions}

The RHF of the ATFT models related to the exceptional Kac-Moody algebras $\mathbf{E}_{6}^{(1)}$ and $\mathbf{E}_{7}^{(1)}$ are constructed. These models generalize the ones in [6] since they contain two types of fields $\vec{q}_{+}(x, t)$ and $\vec{q}_{-}(x, t)$ with different properties with respect to the involution $\mathcal{C}$. The models in [6] contain only fields invariant with respect to $\mathcal{C}$.

We outlined the derivation of the Hamiltonian properties through the classical $R$-matrix approach.

\section{Acknowledgments}

One of us (GGG) thanks the organizing committee of the Sixth International Conference "Symmetry in Nonlinear Mathematical Physics" for the scholarship and for the warm hospitality in Kyiv. The present paper is the written version of the talk delivered by GGG at this conference. The work of GGG is supported by the Bulgarian National Scientific Foundation Young Scientists Scholarship for the project "Solitons, Differential Geometry and Biophysical Models". We also acknowledge support by the National Science Foundation of Bulgaria, contract No. F-1410. We also thank an anonymous referee for careful reading of the manuscript and for useful suggestions.

[1] Gerdjikov V.S., Kyuldjiev A., Marmo G., Vilasi G., Complexifications and real forms of Hamiltonian structures, Eur. Phys. J. B Condens. Matter Phys., 2002, V.29, 177-182.

Gerdjikov V.S., Kyuldjiev A., Marmo G., Vilasi G., Real Hamiltonian forms of Hamiltonian systems, Eur. Phys. J. B Condens. Matter Phys., 2004, V.38, 635-649; nlin.SI/0310005.

[2] Mikhailov A.V., The reduction problem and the inverse scattering problem, Phys. D, 1981, V.3, 73-117.

[3] Mikhailov A.V., Olshanetzky M.A., Perelomov A.M., Two-dimensional generalized Toda lattice, Comm. Math. Phys., 1981, V.79, 473-490.

[4] Olive D., Turok N., Underwood J.W.R., Solitons and the energy-momentum tensor for affine Toda theory, Nucl. Phys. B, 1993, V.401, 663-697.

Olive D., Turok N., Underwood J.W.R., Affine Toda solitons and vertex operators, Nucl. Phys. B, 1993, V.409, 509-546; hep-th/9305160.

[5] Evans J., Complex Toda theories and twisted reality conditions, Nucl. Phys. B, 1993, V.390, $225-250$.

Evans J., Madsen J.O., On the classification of real forms of non-Abelian Toda theories and $W$-algebras, Nucl. Phys. B, 1999, V.536, 657-703; Erratum, Nucl. Phys. B, 1999, V.547, 665-666; hep-th/9802201.

Evans J., Madsen, J.O., Real form of non-Abelian Toda theories and their $W$-algebras, Phys. Lett. B, 1996, V.384, 131-139; hep-th/9605126.

[6] Khastgir S.P., Sasaki R., Instability of solitons in imaginary coupling affine Toda field theory, Prog. Theor. Phys., 1996, V.95, 485-501; hep-th/9507001.

Khastgir S.P., Sasaki R., Non-canonical folding of Dynkin diagrams and reduction of affine Toda theories, Prog. Theor. Phys., 1996, V.95, 503-518; hep-th/9512158.

[7] Gerdjikov V.S., Algebraic and analytic aspects of $N$-wave type equations, Contemp. Math., 2002, V.301, 2002, 35-68. 
[8] Gerdjikov V.S., Analytic and algebraic aspects of Toda field theories and their real Hamiltonian forms, in Bilinear Integrable Systems: from Classical to Quantum, Continuous to Discrete, Editors L. Faddeev, P. van Moerbeke and F. Lambert, NATO Science Series II: Mathematics, Physics and Chemistry, Vol. 201, Dordrecht, Springer, in press.

[9] Gerdjikov V.S., Grahovski G.G., On reductions and real Hamiltonian forms of affine Toda field theories, J. Nonlinear Math. Phys., 2005, V.12, suppl. 2, 153-168.

[10] Bogoyavlensky O.I., On perturbations of the periodic Toda lattice, Comm. Math. Phys., 1976, V.51, 201-209.

[11] Damianou P.A., Multiple Hamiltonian structure of the Bogoyavlensky-Toda lattices, Rev. Math. Phys, 2004, V.16, 175-241.

[12] Gerdjikov V.S., Evstatiev E.G., Ivanov R.I., The complex Toda chains and the simple Lie algebras - solutions and large time asymptotics, J. Phys. A: Math. Gen., 1998, V.31, 8221-8232; solv-int/9712004.

Gerdjikov V.S., Evstatiev E.G., Ivanov R.I., The complex Toda chains and the simple Lie algebras - solutions and large time asymptotics - II, J. Phys. A: Math. Gen., 2000, V.33, 975-1006; solv-int/9909020.

[13] Gerdjikov V.S., $Z_{N}$-reductions and new integrable versions of derivative nonlinear Schrödinger equations, in Nonlinear Evolution Equations: Integrability and Spectral Methods, Editors A.P. Fordy, A. Degasperis and M. Lakshmanan, Manchester University Press, 1981, 367-379.

[14] Gerdjikov V.S., Grahovski G.G., Ivanov R.I., Kostov N.A., N-wave interactions related to simple Lie algebras. $\mathbb{Z}_{2}$-reductions and soliton solutions, Inverse Problems, 2001, V.17, 999-1015; nlin.SI/0009034.

[15] Beals R., Coifman R.R., Scattering and inverse scattering for first order systems, Comm. Pure Appl. Math., 1984, V.37, 39-90.

[16] Gerdjikov V.S., Yanovski A.B., Completeness of the eigenfunctions for the Caudrey-Beals-Coifman system, J. Math. Phys., 1994, V.35, 3687-3725.

[17] Humphreys J.E., Reflection groups and Coxeter groups, Cambridge Univ. Press, 1992.

[18] Drinfel'd V., Sokolov V.V., Lie Algebras and equations of Korteweg-de Vries type, Sov. J. Math., 1985, V.30, 1975-2036.

[19] Damianou P.A., Multiple Hamiltonian structure for Toda-type systems, J. Math. Phys., 1994, V.35, 55115541.

[20] Helgasson S., Differential geometry, Lie groups and symmetric spaces, Academic Press, 1978.

[21] Kac V.G., Infinite dimensional Lie algebras, Cambridge Univ. Press, 1990.

[22] Sklyanin E.K., Quantum version of the method of inverse scattering problem, Zap. Nauchn. Sem. Leningrad. Otdel. Mat. Inst. Steklov. (LOMI), 1980, V.95, 55-128 (in Russian).

Kulish P.P., Quantum difference nonlinear Schrödinger equation, Lett. Math. Phys., 1981, V.5, 191-197.

[23] Gerdjikov V.S., Vilasi G., The Calogero-Moser systems and the Weyl groups, J. Group Theory Phys., 1995, V.3, 13-20. 